\title{
Evaluation of intraarterial and intravenous cisplatin chemotherapy in the treatment of metastatic osteosarcoma using an orthotopic xenograft mouse model
}

\author{
Bernhard Robl', Sander Martijn Botter ${ }^{1}$, Giovanni Pellegrini², Olga Neklyudova ${ }^{1}$ and Bruno Fuchs ${ }^{1 *}$
}

\begin{abstract}
Background: Osteosarcoma is the most common primary malignancy of bone. Its treatment relies on the administration of neoadjuvant and adjuvant chemotherapy combined with surgery. Alternative to common intravenous (i.v.) administration of chemotherapeutic drugs, clinical studies also evaluated the benefit of intraarterial (i.a.) administrations. However, conflicting results were obtained when both routes of administration of cisplatin (CDDP), a gold standard drug in osteosarcoma treatment, were compared. In order to overcome clinical confounding factors, we evaluated both routes of drug administration in a mouse model of experimental osteosarcoma.
\end{abstract}

Methods: We directly compared i.v. versus i.a. drug infusions of cisplatin (CDDP), in an orthotopic xenograft mouse model of metastatic osteosarcoma. We performed tumor monitoring using caliper and micro computed tomography and measured tumor perfusion using laser speckle contrast imaging. Histopathological changes were evaluated using hematoxylin and eosin staining as well as immunohistochemistry (cleaved PARP-1, CD31, HIF-1a).

Results: First, an effective concentration of $4 \mathrm{mg} / \mathrm{kg}$ i.a. CDDP was determined that significantly reduced primary tumor volume. We used this concentration of i.a. CDDP and compared it to infusions of i.v. CDDP. Systemic (i.v.) CDDP only showed minor suppression of tumor growth whereas local (i.a.) CDDP strongly inhibited tumor growth and destruction of cortical bone in the tumor-bearing hind limb. Inhibition of tumor growth was linked to a reduced blood perfusion and resulted in increased amounts of tumor necrosis after i.a. CDDP. After treatment with i.a. CDDP, remaining viable tumor tissue responded by increasing expression of HIF-1a. Side effects due to administration of CDDP were minor, showing no differences in kidney damage between i.v. and i.a. CDDP. However, increased epidermal apoptosis in the foot was an indirect marker for locally increased concentrations of CDDP.

Conclusions: Our findings demonstrate the great potential of local administration of cytotoxic chemotherapeutics, such as CDDP. Consequently, we provide a preclinical basis for a renewed interest in the clinical use of i.a. chemotherapy in osteosarcoma therapy.

Keywords: Intraarterial, Cisplatin, Osteosarcoma, Intravenous, Metastasis

\footnotetext{
* Correspondence: research@balgrist.ch

${ }^{1}$ Laboratory for Orthopedic Research, Department of Orthopedics, Balgrist

University Hospital, Forchstrasse 340, Zurich 8008, Switzerland

Full list of author information is available at the end of the article
} 


\section{Background}

Bone cancers are among the deadliest cancers in adolescents, with osteosarcoma as its most common representative $[1,2]$. Subsequent to the introduction of chemotherapy in the early 1970s, 5-year survival rates of osteosarcoma patients with localized disease increased from $20 \%$ to above $60 \%$ [1, 3]. Standard of care for osteosarcoma patients currently includes systemic, intravenous (i.v.) administration of neoadjuvant chemotherapy, combined with surgical resection of the primary tumor, followed by adjuvant chemotherapy. However, 5-year survival rates plateaued at $60 \%$ and survival rates of patients with metastatic disease remained unchanged at a low 20-30\% until today [3].

In osteosarcoma treatment, the use of neoadjuvant chemotherapy is considered valuable, yet without directly improving event-free survival compared to immediate surgery [4]. Especially pathologic analysis of the tumor response has important prognostic value. Good responders (i.e. $90 \%$ or greater tumor necrosis) achieve up to two times better survival rates compared to patients with poor histologic responses [5-7]. In addition, neoadjuvant chemotherapy reduces tumor volumes prior to surgical resection, facilitating limb sparing procedures [8]. Therefore, neoadjuvant chemotherapy with highly cytotoxic drugs in osteosarcoma is commonly accepted as standard of care. One of the chemotherapeutics always included in today's osteosarcoma treatment regimens is cisplatin (Cis-diamminedichloroplatinum, CDDP). However, its use is limited by systemic toxicities such as ototoxicity and nephrotoxicity $[9,10]$.

Therefore, local, yet controlled application of CDDP may be advantageous. One way to achieve this goal is by local drug administration via the tumor feeding artery. Such intraarterial (i.a.) drug administrations were already successfully performed in the 1980s. These studies confirmed a higher bioavailability of the drug after i.a. infusion of CDDP [11-13], explaining superior histological response rates in osteosarcoma [14-16]. For instance, Wilkins et al. achieved a good response in $87 \%$ of the patients if patients with localized osteosarcomas were treated with i.a. CDDP and i.v. doxorubicin [14], compared to only $41 \%$ in similar patient cohorts where i.v. CDDP and i.v. doxorubicin were used [17], and $71 \%$ in case of a three/four-drug regimen comprising methotrexate [6, 17-21]. In addition to better response rates, studies from the St. Luke's Medical Center achieved 10year survival rates of between 82 and $93 \%$ using i.a. CDDP [14, 22]. These survival rates compare favorably to other studies with maximum 10-year survival rates of, at best, $64 \%$ with an i.v. two-drug regimen [17, 23] or up to $70 \%$ with an i.v. three/four-drug regimen [3, 24]. Similarly, canine osteosarcoma patients showed superior responses when CDDP was infused via the tumor feeding artery compared to i.v. infusions [25].

Although these results demonstrate a clear added value of i.a. CDDP in osteosarcoma treatment, a clinical trial comparing both routes of CDDP administration was unable to show a benefit of i.a. chemotherapy [10]. This discrepancy might be explained by the design of the study, dose adaptations, administration of multiple drugs (standard of care) or its multi-institutional approach. In another study comparing i.a. versus i.v. CDDP, superior tumor responses with i.a. CDDP were only seen in the context of a three-drug regimen, and not as part of a four-drug regimen [20]. However, tumor response rates with the three-drug regimen comprising i.a. (77\%) were similar to the rates found with a fourdrug regimen $(81 \%)$.

In summary, these studies demonstrate the difficulty of evaluating the "true" efficacy of i.a. CDDP due to confounding factors such as administration of different combinations of chemotherapeutics and the large difference in reported survival rates (between $50 \%$ and $71 \%$ already for i.v. CDDP) per treatment center [6, 26]. In addition, tumor heterogeneity and side effect management make it difficult to reliably interpret the results of trials comparing both methods in a clinical setting. In this study, encouraged by the initial promising clinical benefits of i.a. chemotherapy, we investigated, under experimentally controlled conditions, the effects of local (i.a.) versus systemic (i.v.) CDDP in a preclinical mouse model of osteosarcoma.

\section{Methods}

Cell culture and transduction

Human OS 143B cells (CRL-8303) were obtained from American Type Culture Collection (ATCC, USA) and cultured in DMEM (4.5 g/L glucose)/HamF12 (1:1) medium (Invitrogen, USA) supplemented with $10 \%$ heat-inactivated fetal calf serum at $37{ }^{\circ} \mathrm{C}$ in a humidified atmosphere containing $5 \% \mathrm{CO}_{2}$. Previously, 143B cells were transduced with the $L a c Z$ gene [27]. In this study, $143 \mathrm{~B} /$ LacZ cells were additionally infected with retroviral particles containing the mCherry sequence integrated into a pQCIXH backbone, similar as described elsewhere [28]. The original mCherry-containing pcDNA3.1 plasmid was a kind gift from Prof. M. Rudin (Institute of Biomedical Engineering, University and ETH Zurich). After transduction, 143B cells were selected in tissue culture medium with $1200 \mu \mathrm{g} / \mathrm{ml}$ of G418 (Merck, Germany) and $400 \mu \mathrm{g} / \mathrm{ml}$ of hygromycin (Merck, Germany) to stably express LacZ and mCherry.

\section{Animal care}

Female 8-week-old severe combined immunodeficiency mice (CB17/Icr-Prkdc scid/Crl; Charles River Laboratories, 
Germany) were maintained in enriched individually ventilated cages with light/dark-cycles of 12 h/12 h. After delivery, animals were kept for at least a week without any interventions. Food and water was provided to the mice ad libitum. Animal care and experimental procedures were in accordance with the institutional guidelines and approved by the Ethics Committee of the Veterinary Department, Canton of Zurich, Switzerland (License Number 64/2013).

\section{Orthotopic tumor induction in mice}

143B cells were grown to subconfluence, detached with Trypsin/PBS/0.05 \% EDTA, resuspended in PBS/0.05\% EDTA and kept on ice until injected. Before tumor cell injections (TCIs), mice were anesthetized using injection anesthesia. TCIs into left hind limbs were performed similar to as described elsewhere [29]. Briefly, holes were pre-drilled into the medullar cavity of left tibias using sterile needles, before $10^{5} 143 \mathrm{~B}$ cells were injected. After TCIs, mice were monitored weekly for development of primary tumors (see below). Once mice started limping due to the tumor burden, $0.1 \mathrm{mg} / \mathrm{kg}$ of intraperitoneal (i.p.) Buprenorphine (Temgesic; Reckitt Benckiser, UK) was given twice daily. At the end of the study, mice were sacrificed and lung metastases were counted as described [28].

\section{Tumor monitoring}

\section{Primary tumor monitoring}

After TCIs, mice were monitored weekly using caliper and fluorescence measurements, similar as described [28]. Once human 143B osteosarcoma cells established measureable primary tumors (unambigous mCherry signal and a volume greater than $25 \mathrm{~mm}^{3}$ ), drug treatment was started. mCherry tumor fluorescence was measured using an IVIS Lumina XR imaging system (Caliper Life Sciences, Inc., USA) and quantified with Living Image v3.1 software (Xenogen Corporation, USA).

\section{Micro computed tomography}

Micro computed tomography (microCT) using a SkyScan1176 microCT system (SkyScan/Bruker, Billerica, USA) equipped with a $0.5 \mathrm{~mm}$ aluminum filter was conducted to yield high-resolution tomographs of mouse hind limbs. Scans were obtained from each animal at the end of the study at a working source voltage of $50 \mathrm{kV}$ and a source current of $500 \mu \mathrm{A}$ yielding a final image pixel size of $17.7 \mu \mathrm{m}$. Frame averaging of three and exposure times of $210 \mathrm{~ms}$ per projection were set. Each shot required a source rotation step of $0.7^{\circ}$ yielding scan times of approximately $8 \mathrm{~min}$ per mouse. Postacquisition three-dimensional image reconstitution was done in NRecon software v1.6.9.18 (Skyscan/Bruker, USA). Reconstituted images were segmented and bone volumes were calculated using CTAn v1.13.11.0 (Skyscan/Bruker, USA). For calculation of bone and tumor volumes, the region between the distal end of the patella ("start of selection") and the bifurcation of tibia and fibula ("end of selection") was used. Bone volumes were calculated using the following formula: $\Delta$ cortical bone volume $=$ bone volume tumor-limb - bone volume healthy-limb $_{\text {. }}$ Three-dimensional images of the mouse tibias were made in Ctvox v.2.7.0 (Skyscan/Bruker, USA).

\section{Drug infusions}

After induction anesthesia with $5 \%$ isoflurane (Forane; AbbVie, Inc., USA), anesthesia was maintained with $2 \%$ isoflurane during drug infusions. Mice were kept warm on a heating mat throughout the procedure. Intravenous infusions were performed via the tail vein using a $30 \mathrm{G}$ needle attached to a polyethylene catheter (Portex; Smiths Medical, Inc., USA) under control of a syringe pump (Legato; WPI, Inc., USA). Intraarterial infusions were performed similarly as described [30]. Briefly, after revealing the femoral artery proximal to the intratibial tumor, the femoral nerve and the femoral vein were protected by inserting a nitrile strip. Subsequently, the femoral artery was cut and in-house-made, polyethylene catheters were inserted and manually held in place. Drug ( 2 or $4 \mathrm{mg} / \mathrm{kg}$ CDDP; Sandoz, Austria, in $0.9 \% \mathrm{NaCl}$; B. Braun Medical, Inc., Germany, containing $0.8 \%$ patent blue V; Guerbet, France) or vehicle $(0.9 \% \mathrm{NaCl}$ containing $0.8 \%$ patent blue $\mathrm{V}$ ) alone were infused in a total volume of $350 \mu \mathrm{l}$ within 2 min under control of a syringe pump (Legato; WPI, Inc., USA) for three times (every $72 \mathrm{~h}$ ). All manipulations were performed under a stereo microscope (SZX 10; Olympus, Inc., Japan) placed in a sterile working environment. Success of the infusion was controlled through observing the distribution of the blue dye across the hind limb. After removal of the catheter, slight pressure was applied to the injection site in order to prevent bleeding and the site of surgery was flushed with $0.9 \% \mathrm{NaCl}$. The wound was closed with nondegradable silk sutures (7-0 silk; B. Braun Medical, Inc.) in an intermittent pattern. Surgical procedures for an individual i.a. drug infusion took on average $52 \mathrm{~min}$.

In total, two studies were performed: 1) a "dose establishment study" to identify an effective concentration of i.a. CDDP $(\mathrm{N} \geq 4)$, and 2) a "comparison study" (4 mg/kg i.a. $\operatorname{CDDP}(N=11)$ or i.a. vehicle $(N=6)$ versus $4 \mathrm{mg} / \mathrm{kg}$ i.v. CDDP $(N=6)$ or i.v. vehicle $(N=6))$. Overall, i.a. infusions were tolerated well, nevertheless, one mouse treated with $2 \mathrm{mg} / \mathrm{kg}$ i.a. CDDP and two mice treated with $4 \mathrm{mg} / \mathrm{kg}$ i.a. CDDP were sacrificed prematurely during the "establishment study", due to excessive (>15 \%) body weight loss. Throughout the "comparison study", one mouse of the i.v. vehicle-group had to be sacrificed due excessive body weight loss. Two mice from the group of i.v. CDDP dropped out, one during injection, another one was sacrificed due to excessive body 
weight loss. One mouse of the i.a. vehicle group died during the third surgery for unknown reasons. Only one mouse from the i.a. CDDP group dropped out of the study, after being found dead in the cage for unknown reasons. Drop outs were excluded from the analysis.

\section{Hind limb blood perfusion measurements}

Laser speckle contrast imaging of the hind limbs of mice was conducted using a moorFLPI Full-Field Perfusion Imager (Moor instruments Ltd., UK) while mice were fixed in supine position. Imaging was done under lowlight conditions on a heating pad set to $37{ }^{\circ} \mathrm{C}$. Analysis of perfusion was done using the recorded flux (arbitrary units) images and the moorFLPI Review software v3.0 (Moor instruments Ltd.) by placing regions of interest (ROIs) where the primary tumor developed as well as in the corresponding region of the contralateral limb. Flux-ratios were calculated using the following formula: flux-ratio $=$ flux $_{\text {tumor }} /$ flux $_{\text {contralateral }} \times 100 \%$.

\section{Histological and immunohistochemical analysis}

Shortly after euthanasia, primary tumors were cut in equal parts, one snap frozen and the other part decalcified, paraffin-embedded and stained using routine methods. All slides were scanned using a digital slide scanner (NAnoZoomer-XR C12000, Hamamatsu Photonics K.K., Japan) and images were obtained using the corresponding NDP.view2 software. Quantitation of tumor necrosis was conducted using frozen and paraffin-embedded, hematoxylin and eosin (H\&E)stained sections of the tumors, assessing manually the proportion of necrotic tissues versus the total amount of tumor tissue available in the sections. Immunohistochemistry (IHC) was applied on frozen tumor sections to detect apoptotic cells (anti-cleaved PARP1 rabbit monoclonal antibody, \#5625S, Cell Signaling Technology, Inc., USA; 1:50), HIF- $1 \alpha$ (anti-HIF- $1 \alpha$ rabbit polyclonal antibody, NB100479, Novus Biologicals, LLC; USA; 1:500), CD31 (anti-PECAM-1 rabbit polyclonal antibody, sc-1506-R, Santa Cruz Biotechnology, Inc., USA; 1:1000) and Von Willebrand Factor (anti-factor VIII-related antigen (FVIII-Rag) rabbit polyclonal antibody, A0082, DakoAgilent Technologies, Denmark; 1:100). All immunohistochemical stains were performed using a Dako Autostainer (Dako-Agilent Technologies). A minimum of five high power fields (10X magnification in NDP.view2) or if less, the maximum available tissue area were used for analysis using ImageJ v1.47 (U. S. National Institutes of Health).

In the H\&E-stained kidney sections, at least 300 proximal tubules from four randomly selected cortical regions were analyzed by a veterinary pathologist (GP) and a researcher (BR) in a blinded fashion. Tubules exhibiting degenerative changes of the lining epithelial cells such as pyknosis, fragmentation and absence of the nucleus and cytoplasmic hypereosinophilia were counted and normalized to the total number of healthy tubules using ImageJ v1.47 (U. S. National Institutes of Health, USA).

Apoptotic cells in the epidermis of tumor-bearing and tumor-free hind limbs were counted by a pathologist (GP) on the digital scans of the H\&E-stained sections and expressed as average number of apoptotic cells per $\mathrm{cm}$ of skin ( $2 \mathrm{~cm}$ of epidermis evaluated in each limb). Apoptotic keratinocytes (AKs) exhibited a small, strongly basophilic, often fragmented nucleus and a round-up intensely eosinophilic cytoplasm. Apoptosis was confirmed using IHC for cleaved caspase-3 on paraffin-embedded sections (anti-cleaved caspase 3 rabbit monoclonal antibody, \#9664, Cell Signaling Technology, Inc; 1:50).

\section{Statistical analysis}

The results were given as mean \pm standard error of the mean (SEM) unless otherwise stated. If Gaussian distributions were assumed, population means were compared with one-way ANOVA (for analysis of metastases, bone volume, necrosis, IHC stains) or repeated measures two-way ANOVA (for analysis of body weights, tumor volumes, blood perfusion) using Prism 5 v5.01 software (GraphPad Software, Inc., USA) followed by Bonferroni posttests. Using Prism 5, Pearson correlation calculations (HIF-1 $\alpha$ versus CD31) as well as the Kruskal-Wallis test (tubular degeneration) and the Wilcoxon matched pairs test (number of AKs) were performed. Fisher's exact test was calculated using SPSS Statistics v22 (IBM, USA). All statistical tests were 2 -sided and $p<0.05$ was regarded as statistically significant.

\section{Results}

Establishment of an i.a. drug injection model for treating osteosarcoma

First, the concentration of i.a. CDDP that led to a significant reduction in primary tumor growth was identified, which would later on be used in comparison with i.v. CDDP. After orthotopic injection of 143B osteosarcoma cells, different concentrations of CDDP in $\mathrm{NaCl}(0.9 \%)$ vehicle were i.a. infused into the femoral artery of the tumor-bearing limb. Only $4 \mathrm{mg} / \mathrm{kg}\left(30 \pm 11 \mathrm{~mm}^{3}\right)$ and not $2 \mathrm{mg} / \mathrm{kg}\left(128 \pm 30 \mathrm{~mm}^{3}\right)$ of i.a. CDDP resulted in significant retardation of tumor growth compared to the vehicle (180 $\pm 89 \mathrm{~mm}^{3}$; Fig. 1a). Moreover, X-gal staining of tumor cells on the surface of lungs revealed a trend towards a dose-dependent reduction of lung metastases after i.a. CDDP (Fig. 1b). Administration of chemotherapeutics such as CDDP in preclinical models often leads to body weight loss but no significant differences between vehicle, $2 \mathrm{mg} / \mathrm{kg}$ i.a. CDDP and $4 \mathrm{mg} / \mathrm{kg}$ i.a. CDDP were noted (Fig. 1c). Every drug infusion was assessed visually by observing a color change from white to blue of the infused areas after successful infusion (Fig. 1d). Infusion 


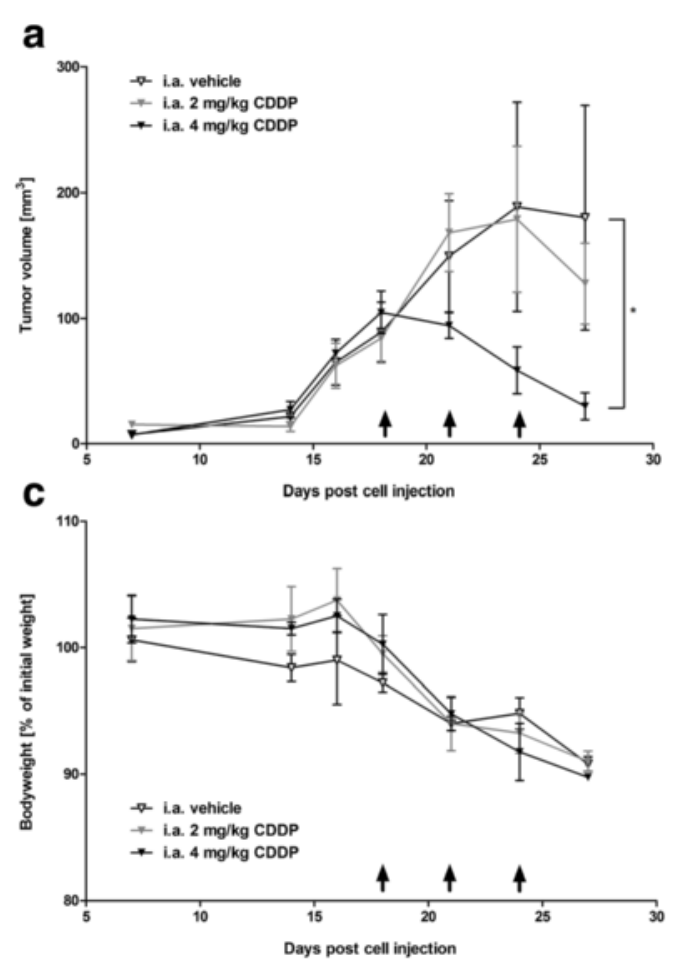

b
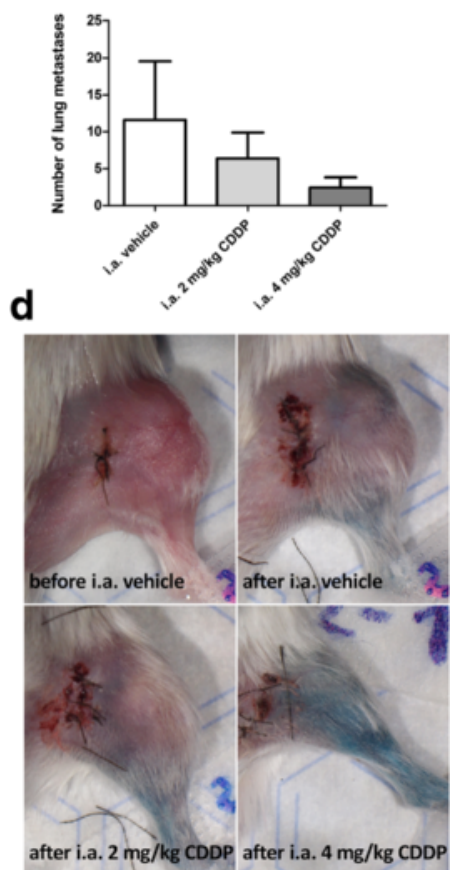

Fig. 1 Identification of an effective concentration of i.a. CDDP. a Tumor volumes after three separate treatments with 0, 2, or 4 mg/ $\mathrm{kg}$ of i.a. CDDP. Tumor volumes were determined by caliper measurements. b Presence of pulmonary metastases after treatment with 0,2 , or $4 \mathrm{mg} / \mathrm{kg}$ of i.a. CDDP. Metastases on the surfaces of lungs were counted ex vivo after $\mathrm{X}$-gal staining. $\mathbf{c}$ Changes in body weight as an indicator for general health of the mice. $\mathbf{d}$ Examples of tumor-bearing hind limbs; before the third infusion of i.a. vehicle (upper left) and after the third successful infusion of i.a. vehicle (upper right), $2 \mathrm{mg} / \mathrm{kg}$ i.a. CDDP (lower left) and $4 \mathrm{mg} / \mathrm{kg}$ CDDP (lower right). The appearance of the blue color across the leg indicated a successful infusion. Days of drug infusion are indicated by black arrows $(\mathbf{\uparrow}){ }^{*} p<0.05$ as compared to the vehicle

quality controls indicated homogeneous dye distribution after three infusions of $4 \mathrm{mg} / \mathrm{kg}$ i.a. CDDP, whereas vehicle and $2 \mathrm{mg} / \mathrm{kg}$ i.a. CDDP yielded inhomogeneous dye distributions within the region of tumor growth.

\section{Osteosarcoma development dependent on the route of CDDP administration}

Next, a comparison between i.v. and i.a. CDDP infusions was conducted. Only i.a. CDDP $\left(88 \pm 31 \mathrm{~mm}^{3}\right)$ inhibited tumor growth and caused regression of primary tumors, while tumors continued to grow in all other treatment groups (tumor volumes at 27 days post tumor cell injection: i.v. CDDP: $307 \pm 25 \mathrm{~mm}^{3}$; i.a. vehicle: $375 \pm 73 \mathrm{~mm}^{3}$; i.v. vehicle: $491 \pm 44 \mathrm{~mm}^{3}$; two-way ANOVA: $p<0.0001$; Fig. 2a). One day prior to sacrifice, mice were subjected to microCT scans. Tumor volumes measured within the resulting tomographs confirmed caliper measurements and yielded significantly smaller final tumor volumes in the group receiving i.a. CDDP $\left(54 \pm 35 \mathrm{~mm}^{3}\right)$ compared to volumes measured in other treatment groups (i.v. CDDP: $297 \pm$ $29 \mathrm{~mm}^{3}$; i.a. vehicle: $286 \pm 58 \mathrm{~mm}^{3}$; i.v. vehicle $479 \pm$ $34 \mathrm{~mm}^{3}$; ANOVA: $\left.p<0.0001\right)$. Osteosarcoma is known to be associated with pathological bone remodelling and increased fracture risk, and thus, the structural integrity of the bone influences the quality of life of osteosarcoma patients. 143B cell-derived osteosarcomas were shown to behave mostly osteolytic in vivo and loss of cortical bone correlates with increasing tumor volume. Accordingly, administration of i.a. CDDP ( $87 \pm 5 \%$ of initial bone volume (before treatment)) led to the smallest loss of cortical bone compared to i.v. vehicle $(75 \pm 3 \%)$, i.a. vehicle $(68 \pm 6 \%)$ and i.v. CDDP (51 $\pm 2 \%$; ANOVA: $p<0.0001$; Fig. $2 b, c)$.

Finally, X-gal staining of lacZ tagged cells on the surface of lungs collected during necropsy (Fig. 2d) showed that systemic i.v. CDDP had no significant effect on metastatic spread towards the lung compared to i.v. vehicle control (i.v. CDDP: $202 \pm 15$; i.v. vehicle: $218 \pm 41$ ). In contrast, i.a. CDDP significantly reduced the number of lung metastases (i.a CDDP: $82 \pm 42$; i.a vehicle: $695 \pm$ 300; ANOVA: $p<0.001$; Fig. 2e). Of note, a nonsignificant, but on average higher amount of metastases was found in i.a. vehicle versus i.v. vehicle group.

\section{Effect of CDDP treatment on tumor blood perfusion}

Tumor-associated vasculature was assessed in vivo via blood perfusion measurements. Primary tumor growth 


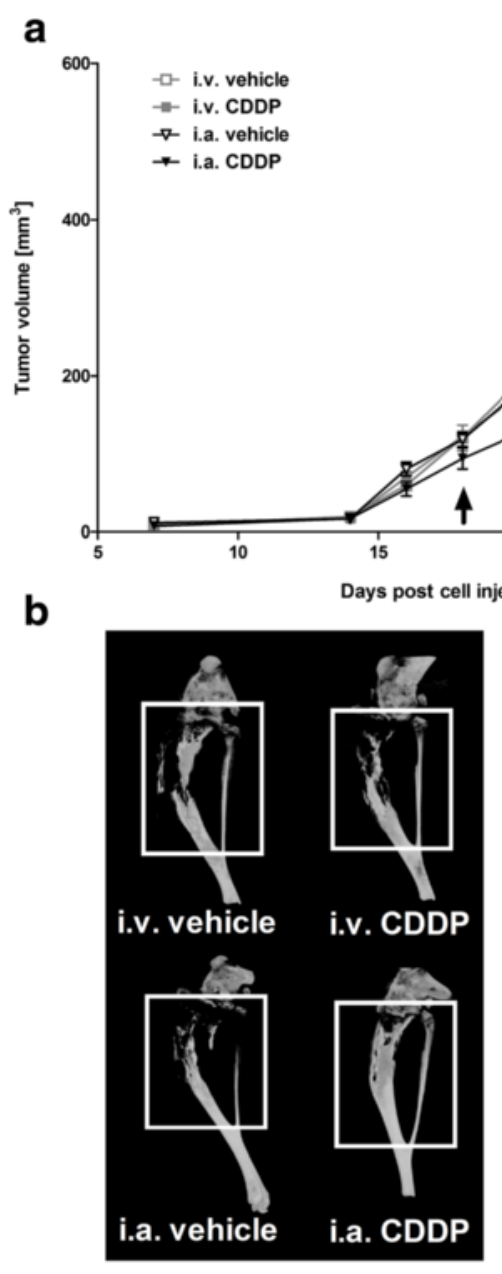

d
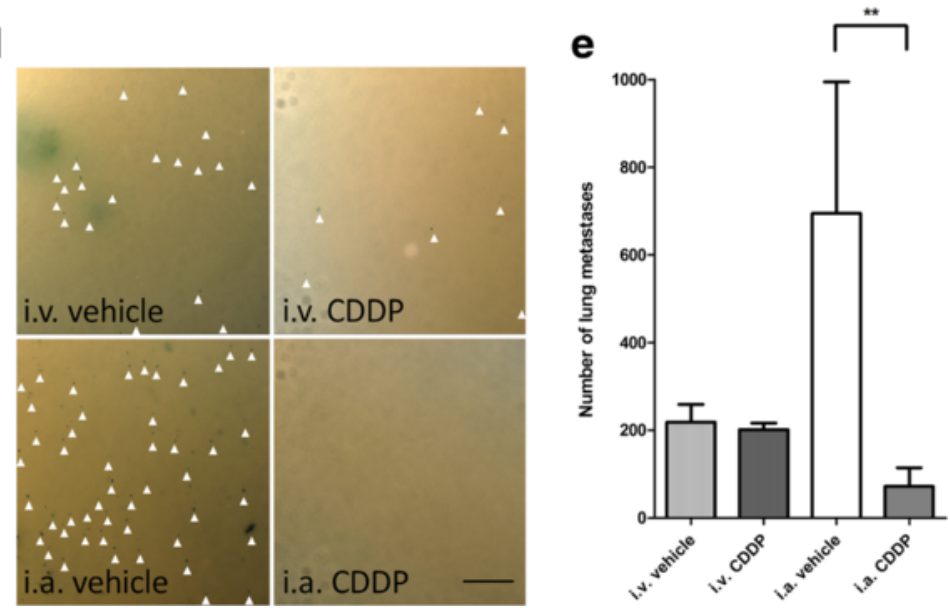

Fig. 2 Effects of different routes of CDDP administration on osteosarcoma development. a Tumor volumes after treatment with vehicle or 4 mg/ $\mathrm{kg}$ CDDP, both given i.v. and i.a.. Tumor volumes were determined by caliper measurements. Days of drug infusion are indicated by black arrows (4). Two-way ANOVA: significant differences are only indicated for day 27. b Representative microCT scans of tumor-bearing bone. White squares mark the areas between the distal end of the patella and the bifurcation of tibia and fibula, which was used for quantification of differences in cortical bone. c Quantitation of differences in cortical (mineralized) bone volume as determined by microCT measurements. $\mathbf{d}$ Representative images of X-gal stained lung metastases. White arrowheads ( $\Delta$ ) indicate lac $Z^{+}$lung metastases. Scale bar corresponds to $500 \mu \mathrm{m}(4 \mathrm{X})$. e Quantitation of number of pulmonary metastases on the entire lung surface. ${ }^{*} p<0.05 ;{ }^{* *} p<0.01 ;{ }^{* * *} p<0.001$ as compared to the indicated treatment 
induced an increase in perfusion of the tumor-bearing limbs compared to the contralateral control limbs (Fig. 3a). Following i.a. CDDP, a significant decrease in perfusion compared to i.v. CDDP or i.a. vehicle was observed (two-way ANOVA: $p<0.05$; Fig. $3 \mathrm{~b}$ ). Interestingly, the largest reduction in perfusion were detected after i.a. CDDP infusions. At the end of the study, perfusion of the i.a. CDDP-treated limbs was close to physiological values, similar to the contralateral knee region. However, areas formerly infiltrated by osteosarcomas appeared poorly perfused, indicating the occurrence of ischemic tumor necrosis (e.g. Fig. 3a: i.a. CDDP).

\section{Histologic response to CDDP chemotherapy}

Assessment of tumor necrosis after neoadjuvant chemotherapy is an established endpoint to evaluate the response to treatment in osteosarcoma patients. Figure 4a illustrates representative examples from each treatment group which were used for the analysis of tumor necrosis. In case of two animals treated with i.a. CDDP, no tumor tissue could be found on cross sections of the tumorbearing limb, indicating a strong anti-tumor effect (100\% of tumor necrosis was assumed). The largest mean tumor necrosis was detected after i.a. CDDP $(68 \pm 12 \%)$ compared with i.a. vehicle $(32 \pm 8 \%)$, i.v. CDDP $(17 \pm 2 \%)$ or i.v. vehicle ( $21 \pm 3 \%$, ANOVA: $p<0.01$; Fig. $4 \mathrm{~b})$. According to Salzer-Kuntschik, a good responder is defined by more than $90 \%$ tumor necrosis [31]. With i.a. CDDP, a total of five $(45 \%)$ good responses was achieved, whereas no good responses were detected with i.a. vehicle, i.v. vehicle or i.v. CDDP (Fisher's exact test: $p<0.01$; Additional file 1). Tumor cell death in the H\&E-stained sections consisted of multifocal to coalescing, variably sized areas of necrosis: these areas which are, to varying extents, inherent to any rapidly growing tumor (i.e. after i.v. vehicle) are likely indicative of ischemic cell death. a
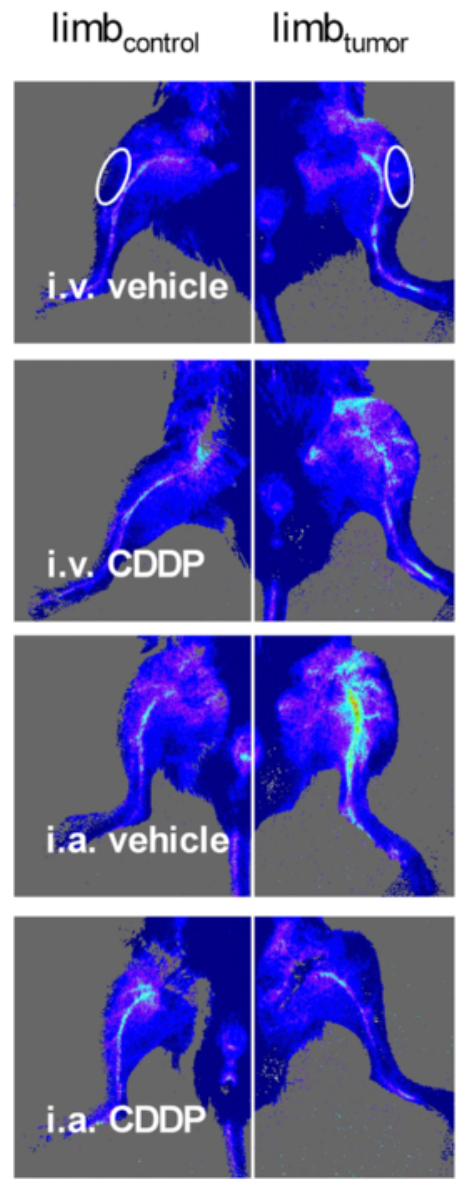

b

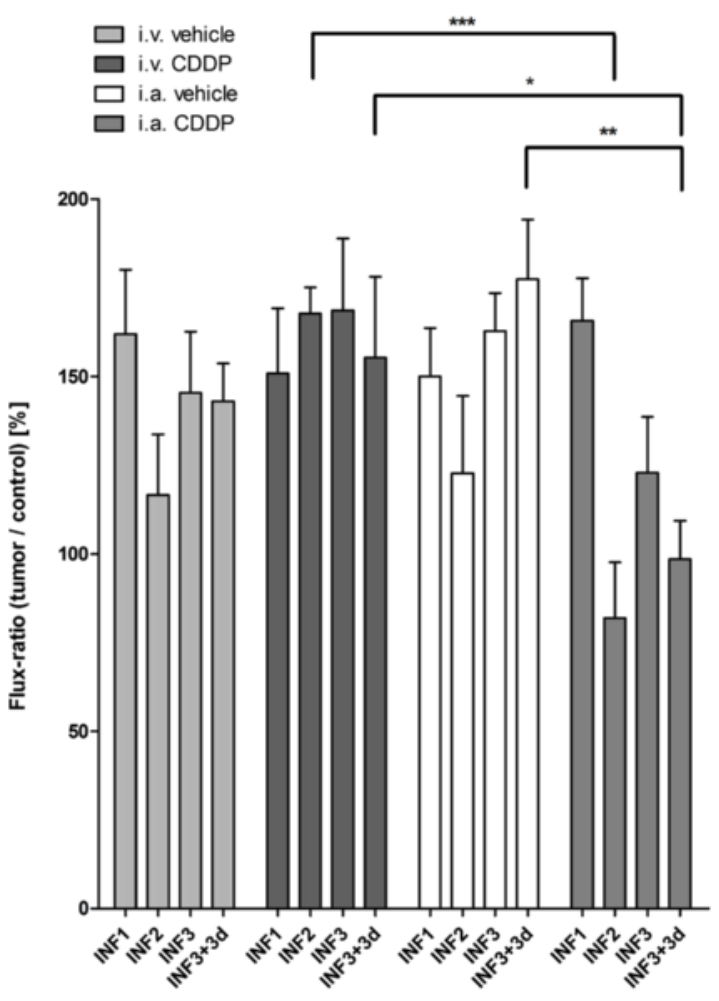

Fig. 3 Changes in hind limb blood perfusion during the treatment period. a Representative images of perfusion measurements of the knee region from each treatment group at the end of the study (27 days post tumor cell injection). Images in the left column illustrate healthy contralateral limbs. Perfusion images in the right column illustrate tumor-bearing limbs. Circular ROls (only indicated for "i.v. vehicle") were used for measurements. $\mathbf{b}$ Flux-ratios of knee regions during the entire treatment period for individual treatment groups. Labeling of the $x$-axis indicates the respective measurement times: INF1/2/3: immediately prior to the first/s/third infusion; INF3 + 3d: three days after the final third infusion 


\section{a}

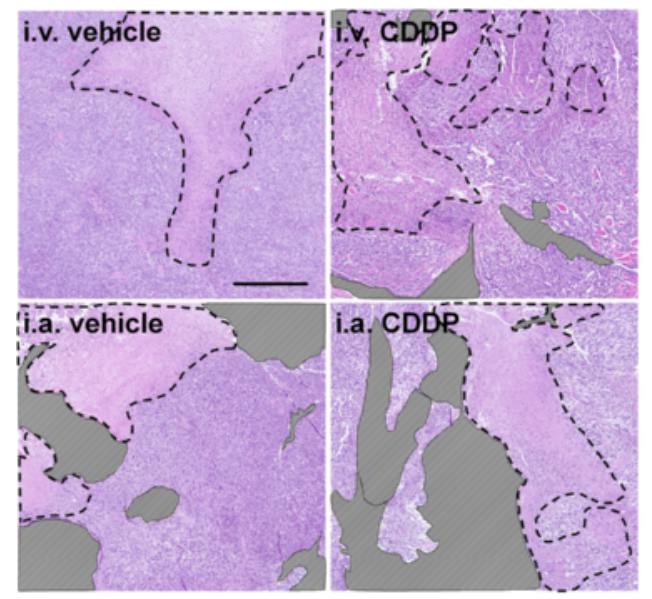

b

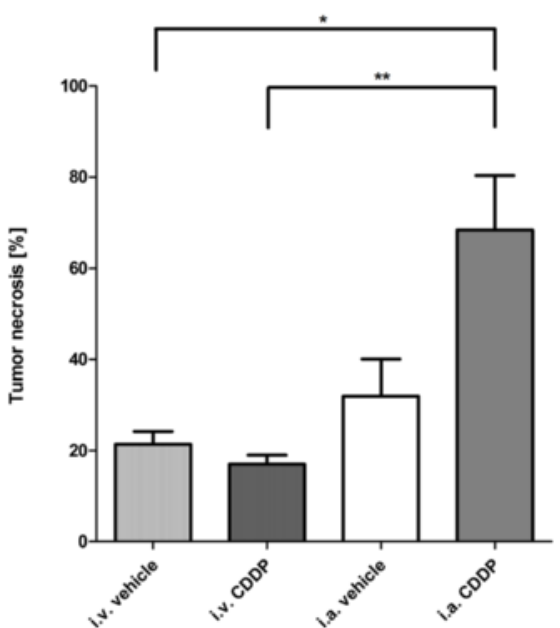

Fig. 4 Histological evaluation of tumor necrosis. a Representative examples of necrotic (black dashed lines) tumor areas are shown for each treatment group. Areas overlaid with a striped pattern were not considered for evaluation (e.g. bone, muscle, absence of tissue). Scale bar corresponds to $500 \mu \mathrm{m}(5 \mathrm{X})$. b Quantitation of tumor necrosis, normalized to the total tumor area which was available for analysis. ${ }^{*} p<0.05$ as compared to the indicated treatment

\section{Influence of different routes of CDDP administration on remaining viable tumor tissue}

Due to the anti-tumor efficacy of i.a. CDDP, smaller areas of viable tumor were evaluated after treatment: i.a. CDDP (median $0.6 \mathrm{~mm}^{2}$; interquartile range $0.1-4.0 \mathrm{~mm}^{2}$ ) compared with i.a. vehicle $\left(7.1 \mathrm{~mm}^{2} ; 3.9-11.3 \mathrm{~mm}^{2}\right)$, i.v. CDDP $\left(10.0 \mathrm{~mm}^{2} ; 7.2 .-12.7 \mathrm{~mm}^{2}\right)$ and i.v. vehicle (11.9 $\left.\mathrm{mm}^{2} ; 11.0-14.8 \mathrm{~mm}^{2}\right)$. Furthermore, two animals from the i.a. CDDP group were excluded from all immunohistochemical analyses involving viable primary tumor because of a total absence of tumor tissue. Within regions of viable tumor, scattered neoplastic cells exhibited morphological features of apoptosis, such as cell shrinkage, nuclear pyknosis and fragmentation, as indicated by immunohistochemical stains for cleaved PARP-1, a marker for chemotherapy-induced apoptosis [32]. However, no significant differences in the number of cleaved PARP $-1^{+}$cells within areas of remaining viable tumor were detected between corresponding vehicle and treatment groups (Fig. 5a).

In order to see if the reduced limb perfusion also resulted in increased hypoxia, expression of HIF-1 $\alpha$, a protein expressed under sub-physiological levels of oxygen, was studied. IHC of HIF- $1 \alpha$ demonstrated intense staining of remaining viable tumor tissue after i.a. CDDP (Fig. 5b). Furthermore, quantitation of HIF1-1 $\alpha$ expression demonstrated a significant increase in HIF- $1 \alpha$ levels in tumors after administration of i.a. CDDP $(2.8 \pm 0.7 \%$ of remaining viable tumor tissue) compared with tumors exposed to i.a. vehicle $(0.6 \pm 0.6 \%)$, i.v. CDDP $(0.4 \pm$ $0.2 \%)$ or i.v. vehicle $(0.3 \pm 0.1 \%$; ANOVA: $p<0.001$; Fig. 5c). High levels of HIF- $1 \alpha$ indicate low oxygen levels, subsequently triggering neovascularization. To this end, IHC for CD31 and factor VIII-related antigen (FVIII-RAg) was performed to characterize and quantify newly formed blood vessels within the neoplasms [33]. Examples of CD31 IHC are shown in Fig. 5d. In all groups, areas of viable tumor contained negligible numbers of FVIII-Rag ${ }^{+}$blood vessels, while the endothelial cells lining large vessels in the skeletal muscle and connective tissue adjacent to the osteosarcomas expressed FVIII-RAg (data not shown). Quantitation of the CD31 ${ }^{+}$ areas within viable tumor tissue indicated a trend towards increased neovascularization after i.a. CDDP $(4.1 \pm 1.5 \%$ of remaining viable tumor tissue) compared with the lower levels observed after i.a. vehicle $(1.8 \pm 0.7 \%)$, i.v. CDDP $(1.6 \pm 0.2 \%)$ or i.v. vehicle treatment $(1.3 \pm 0.2 \%$; Fig. 5e). Furthermore, CD31 IHC significantly correlated with HIF-1 $\alpha$ IHC (Pearson's $r: p<0.01 ; \mathrm{r}=0.62$; Fig. 5f).

\section{Side effects associated with different routes of CDDP administration}

Similar to the "dose establishment study", body weights were measured at regular intervals throughout the "comparison study" (Fig 6a). When comparing CDDP administrations only (i.a. CDDP: $88 \pm 2 \%$ of body weight normalized to the weight at day of tumor cell injection versus i.v. CDDP: $85 \pm 1 \%$ ), no significant difference in body weight development between i.a. or i.v. CDDP administration was demonstrated. In contrast, i.v. CDDP caused a significant drop in body weight compared with i.v. vehicle $(97 \pm 2 \%)$, whereas no difference was found between i.a. CDDP and i.a. vehicle ( $90 \pm 2 \%$; two-way ANOVA: $p<0.0001$; Fig. 6a). 


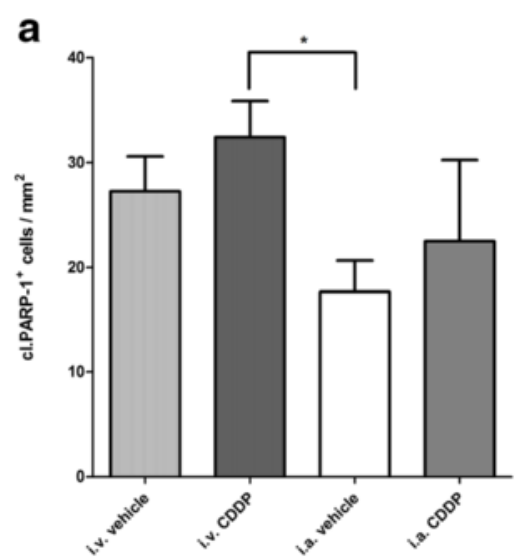

d

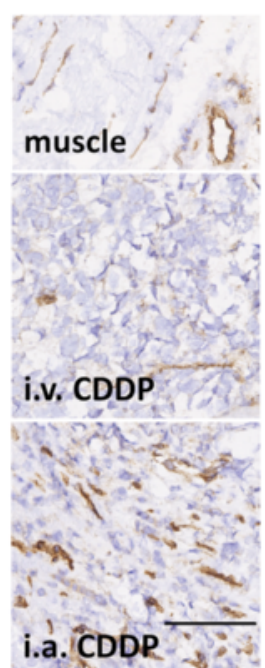

b

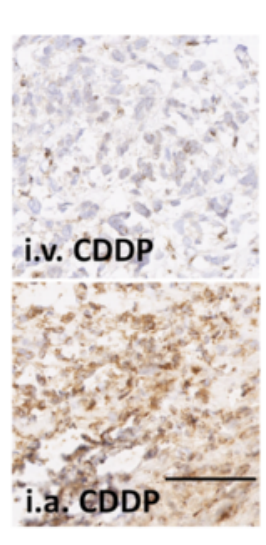

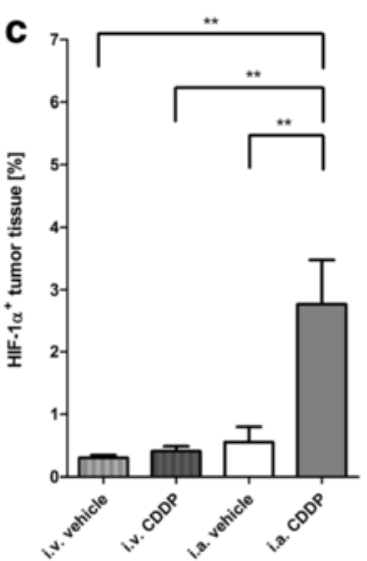

f
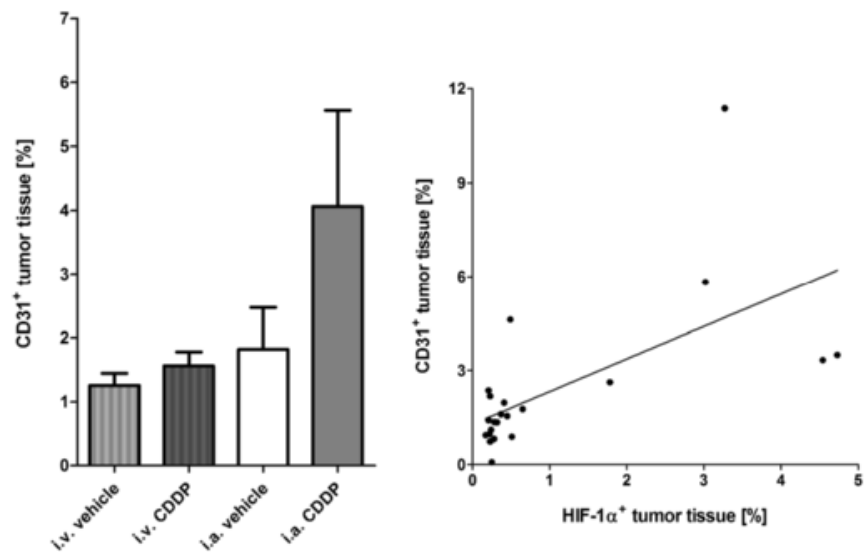

Fig. 5 Effects of different routes of CDDP administration on remaining viable tumor tissue. a Number of cleaved PARP- $1^{+}$tumor cells. PARP- $1^{+}$ tumor cells were only counted within areas of viable tumor tissue. $\mathbf{b}$ Representative images of tumor tissue (i.v. CDDP, i.a. CDDP) stained for HIF-1a (20X). Scale bar corresponds to $100 \mu \mathrm{m}$. c Quantitation of HIF-1a tumor tissue normalized to the entire viable tumor tissue available for evaluation. $\mathbf{d}$ Representative images of healthy adjacent tissue (muscle) and tumor tissue (i.v. CDDP, i.a. CDDP) stained for CD31 (20X), where the upper image shows CD31 expression in the endothelial cells lining capillaries as well as the larger vessels in the skeletal muscle surrounding the tumors, the central image represents CD31 expression after i.v. CDDP and the lower image displays an increase in CD31 expressing cells within the tumor mass after i.a. CDDP. Scale bar corresponds to $100 \mu \mathrm{m}$. e Quantitation of $\mathrm{CD}_{3} 1^{+}$tumor tissue normalized to the entire tumor tissue available for evaluation. $\mathbf{f}$ Correlation of HIF-1a IHC with CD31 IHC (Pearson's $r=0.62$ ). ${ }^{*} p<0.05 ;{ }^{* *} p<0.01$ as compared to the indicated treatment

In general, application of CDDP is limited by a high incidence of severe nephrotoxicity characterized by degeneration or death of the proximal tubule epithelial cells $[34,35]$. In this study, however, no histological abnormality was recognized in the kidneys, except in three mice after i.a. CDDP, which exhibited mild acute tubular degeneration/necrosis, affecting only a small proportion of the renal tubules (Fig. 6b).

To assess whether i.a. CDDP resulted in higher apoptotic rates of non-malignant cells, suggestive of higher local concentrations of CDDP in the operated limb, the number of apoptotic keratinocytes within the skin of treated limbs and contralateral limbs were quantified. Results demonstrated lower numbers of apoptotic keratinocytes (AK) after i.v. vehicle $(0-0.05 \mathrm{AK} / \mathrm{cm}$ of skin; minimum - maximum of tumor bearing limb), i.v. CDDP $(0-0.1 \mathrm{AK} / \mathrm{cm})$ or i.a. vehicle treatment $(0-0.05$ $\mathrm{AK} / \mathrm{cm}$ ), in tumor-bearing limbs as well as in the corresponding contralateral limb (Fig. 6c). In contrast, significantly higher numbers of AKs were found after the administration of i.a. CDDP $(0.35-7.6 \mathrm{AK} / \mathrm{cm})$, yet in the tumor-bearing limb only (Wilcoxon matched pair test: $p<0.01$; Fig. $6 \mathrm{c}$ ).

\section{Discussion}

In this study, we present the successful establishment of i.a. drug administrations in a mouse model of experimental orthotopic osteosarcoma. Using CDDP as a gold 


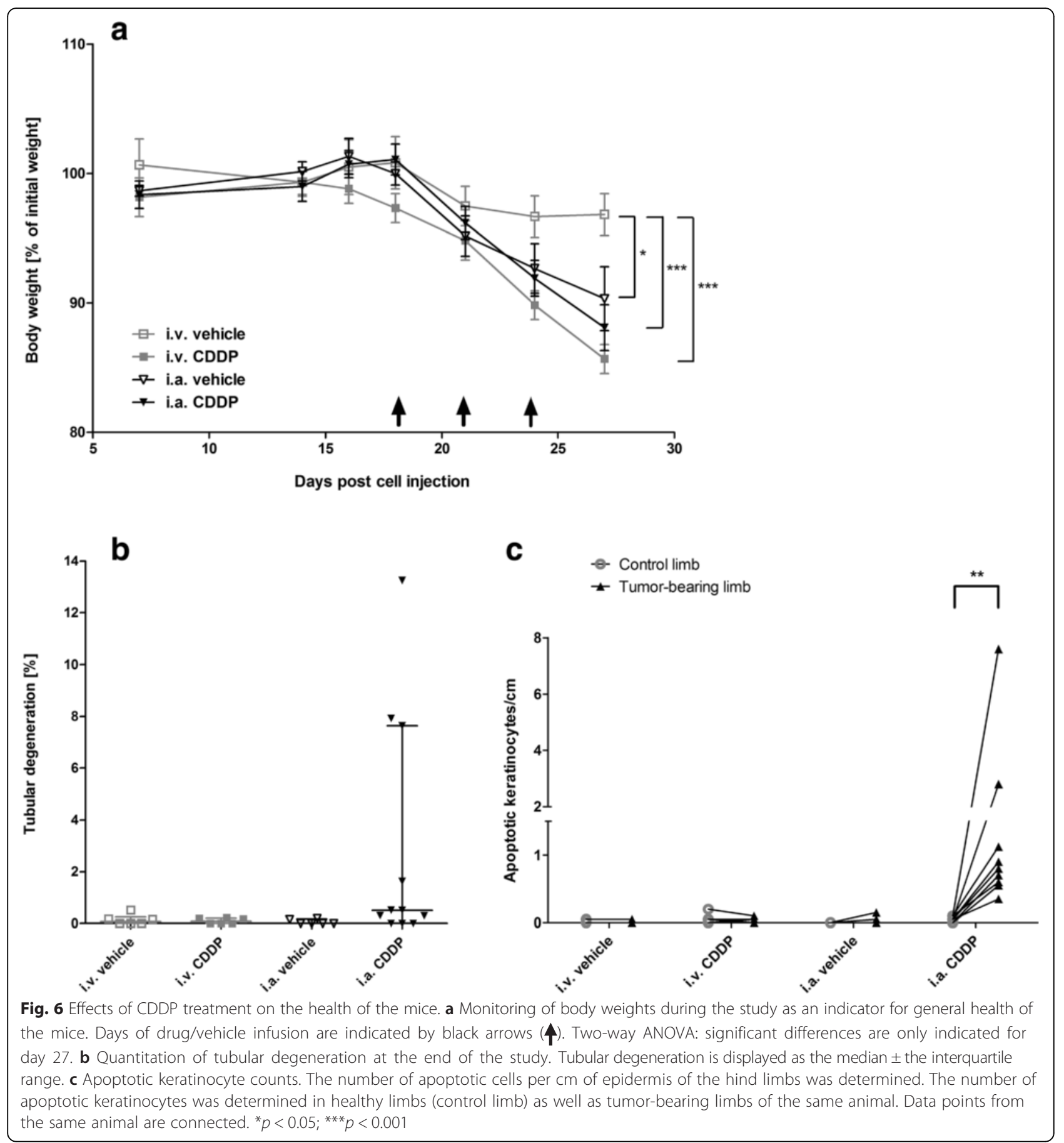

standard drug for osteosarcoma treatment, we showed that our setup of i.a. drug infusions is feasible and that primary and systemic disease could be inhibited in a concentration-dependent manner. Moreover, we demonstrated that i.a. CDDP is more effective in inhibiting osteosarcoma progression than equivalent concentrations of i.v. CDDP, as indicated by smaller primary tumor volumes, decreased destruction of cortical bone as well as decreased numbers of lung metastases.
Increased anti-tumor efficacy of i.a. CDDP infusions was also confirmed by histological analyses, where we demonstrated increased levels of tumor necrosis. Decreased tumor blood perfusion and increased hypoxia of the neoplasms after i.a. CDDP administration was demonstrated and explains, at least partially, the superior efficacy of localized CDDP delivery. Finally, we showed that i.a. CDDP causes increased levels of apoptotic keratinocytes in the epidermis of tumor-bearing limbs, while 
other systemic side effects were similar compared with i.v. CDDP.

Despite the use of larger animal model systems such as dogs [25] or sheep [36], the most commonly used model systems in osteosarcoma research are rodents [37]. To our knowledge, this is the first report of an i.a. infusion model in experimental, orthotopic osteosarcoma in mice, where we could demonstrate superior tumor control as compared to routine i.v. infusions. Hence, our i.a. model can be used as a platform for the investigation of other small molecules whose systemic application is limited by side effects. Especially for osteosarcomas of the limb, easy access to the tumor feeding artery offers a valuable alternative to systemic CDDP infusions. However, i.a. infusions are not limited to tumors of the limb. Another recent study using a mouse model of metastatic brain tumors demonstrated advantages in tumor control with i.a. chemotherapy (internal carotid artery) compared with i.v. chemotherapy (tail vein), similar to our study [38]. This demonstrates that even difficult-to-access tumor entities can be treated with i.a. drug infusions.

Local application of small molecules such as CDDP offers several advantages compared with systemic application. First, higher tumor drug loads can be achieved after infusion of equivalent drug concentrations via the tumor-feeding artery [11], causing greater tumor necrosis [13]. In line with this assumption, we demonstrated greater tumor necrosis after i.a. CDDP compared with i.v. CDDP administration. In contrast to Winkler et al., we were able to compare i.a. versus i.v. routes of administration in the absence of clinical confounding factors such as changes in drug infusion times or stratification of patients (e.g. high-risk only) [10]. In our study, i.a. CDDP not only caused a reduction of the primary tumor volume but also minimized the loss of mineralized bonean indicator for experimental osteosarcoma progression [39]. In contrast, loss of cortical bone was increased after i.v. CDDP treatment compared with i.v. vehicle. Likewise, when sites of bone turnover in dogs were studied, bone remodeling was significantly influenced by the systemic administration of CDDP [40].

Systemic side effects, especially nephrotoxicity, are equal or reduced after local CDDP infusion compared to systemic application without a simultaneous reduction of the systemic potency of the drug $[9,20]$. In our study, most animals showed no signs of nephrotoxicity. However, we found evidence of mild nephrotoxicity, represented by scattered tubular degeneration/necrosis in three animals treated with i.a. CDDP. Renal injury was minor and unlikely to have an impact on kidney function. It is possible that hypovolemia, resulting from blood loss and/or insufficient rehydration after the repeated surgical interventions, exacerbated the observed nephrotoxicity in these animals.
Skin necrosis is another side effect observed in human patients after i.a. administration of CDDP [10, 20]. However, this usually does not lead to complications during the treatment and regenerates well. Likewise, our results indicated that i.a. CDDP led to increased numbers of apoptotic keratinocytes in the tumor-bearing limbs, but not in the healthy contralateral limbs. Elevated numbers of AK may indicate a higher local CDDP concentration in the proximity of the primary tumor and thus, help to explain the superior response after administration of i.a. CDDP compared with i.v. CDDP. Interestingly, some mild chemotherapy-induced toxicities were shown to be associated with improved osteosarcoma patient survival [23].

Consistent with the observations reported by Wilkins et al., where a reduction of the spongy tumor vasculature after i.a. CDDP was detected [14, 22], we also observed a general decrease in perfusion of the tumor region shortly after i.a. CDDP administration. This reduction in tumor perfusion in addition to the higher local CDDP concentration may have further contributed to the regression of the experimental tumors and resulted in a good histological response (as defined by having at least $90 \%$ tumor necrosis) in at least $45 \%$ of the mice. Thus, destruction of the tumor vasculature seems to be a necessity for i.a. CDDP to successfully induce tumor necrosis potentially resulting in a high percentage of good histologic responses [14].

The reduction in tumor perfusion after i.a. CDDP might have caused the remaining viable tumor tissue to react by expressing increased levels of HIF- $1 \alpha$. It is known that constitutively active HIF- $1 \alpha$ induces neovascularization and increased expression of CD31 or VEGF [41-44]. Increased expression of HIF- $1 \alpha$ in osteosarcomas after i.a. CDDP was paralleled by increased microvascular density assessed using IHC for CD31, a marker of immature endothelium. In addition to CD31, consecutive tissue sections were stained for FVIII-RAg, normally found in large, mature vessels [33]. The few scattered FVIII-Rag ${ }^{+}$vascular structures found within the tumors were likely pre-existing and no difference was found among the different groups. In summary, our results indicate a response of the neoplasms towards ischemic damage after i.a. CDDP by increasing HIF-1 $\alpha$ levels and potentially initiating neovascularization.

Physical manipulation of the primary tumor as well as changes of blood perfusion within the primary tumor is known to increase numbers of circulating tumor cells and thus, the risk for the development of metastases $[45,46]$. In our study, this might be reflected by a higher, albeit nonsignificant amount of lung metastases after i.a. vehicle administration, which, following i.a. CDDP, was reduced below amounts following i.v. CDDP. Thus, in addition to improved local tumor control, i.a. 
CDDP also successfully controlled the number of spontaneous lung metastases. This is especially relevant in osteosarcoma, where controlling pulmonary metastases strongly influences patient survival [47-49].

One limitation of our study is the inherent variability due to any surgical procedure. Although the same surgeon performed i.a. drug infusions, the parameters of i.a. infusions varied (e.g. duration of surgery or degree of blood loss). Especially the placement of the catheter is critical for a homogeneous drug distribution in subsequent arterial branches [50] and thus, impacts success of therapy. In general, our study suggests a superior outcome in the chemotherapeutic response after i.a. delivery of CDDP, however, the individual outcomes must be interpreted alongside corresponding toxicokinetic information.

\section{Conclusions}

Taken together, our study demonstrates the potential of i.a. CDDP in a clinically relevant osteosarcoma model. The superior primary tumor control of i.a. CDDP in our study demonstrates the potential of i.a. drug administrations as currently used in some clinics. Despite the greater technical requirements for i.a. drug infusions, we suggest that the potential of i.a. infusions in osteosarcoma treatment should be considered when evaluating (novel) compounds and combinations thereof. Especially for a rare disease such as osteosarcoma, we believe that our intraarterial therapy model can aid in the preclinical assessment of drug efficacy and thus, improve osteosarcoma patient treatment.

\section{Additional file}

Additional file 1: Histological response according to treatment. Groups the histological tumor response according to current clinical criteria for evaluation of tumor necrosis. (DOCX $14 \mathrm{~kb}$ )

\section{Abbreviations}

AK, apoptotic keratinocyte; CD31, cluster of differentiation 31; CDDP, cisplatin; FVIII-RAg, factor VIII related antigen; H\&E, hematoxylin and eosin; HIF-1a, hypoxia inducible factor-1a; i.a, intraarterial; i.p, intraperitoneal; i.v, intravenous; IHC, immunohistochemistry; microCT, micro computed tomography; PARP-1, poly ADP ribose polymerase-1; SCID, severe combined immunodeficiency; TCls: tumor cell injections

\section{Acknowledgments}

We would like to thank Prof. Anja Kipar for fruitful discussions regarding the histological analysis of the tumor material.

\section{Funding}

Our work is supported by the University of Zurich, the Schweizerischer Verein Balgrist (Zurich, Switzerland), the Walter L. \& Johanna Wolf Foundation (Zurich, Switzerland), the Highly Specialized Medicine for Musculoskeletal Oncology program of the Canton of Zurich, the Zurcher Krebsliga (Zurich, Switzerland), the "Kind und Krebs" fund (Zollikerberg, Switzerland), and the Swiss National Science Foundation SNF Nr.310030_149649.

\section{Availability of data and materials}

The datasets supporting the conclusions of this article are included within the article and its additional file.

\section{Authors' contributions}

Conception and design: BR, SMB, BF. Development of methodology: BR, ON, BF. Acquisition of data: BR, GP, SMB, ON. Analysis and interpretation of data: $B R, G P$. Writing, review, and/or revision of the manuscript: BR, SMB, BF. Administrative, technical, or material support (i.e., reporting or organizing data, constructing databases): BR, GP, BF. Study supervision: BF. All authors read and approved the final manuscript.

\section{Competing interests}

The authors declare that they have no competing interests.

\section{Consent for publication}

Not applicable.

\section{Ethics approval and consent to participate}

Animal care and experimental procedures were in accordance with the institutional guidelines and approved by the Ethics Committee of the Veterinary Department, Canton of Zurich, Switzerland (License Number 64/ 2013)

\section{Author details}

'Laboratory for Orthopedic Research, Department of Orthopedics, Balgrist University Hospital, Forchstrasse 340, Zurich 8008, Switzerland. ' Laboratory for Animal Model Pathology, Veterinary Pathology, Vetsuisse Faculty, Zurich, Switzerland.

Received: 12 May 2016 Accepted: 8 July 2016

Published online: 16 July 2016

References

1. Mirabello L, Troisi RJ, Savage SA. Osteosarcoma incidence and survival rates from 1973 to 2004: data from the Surveillance, Epidemiology, and End Results Program. Cancer. 2009;115(7):1531-43.

2. Howlader N, Noone AM, Krapcho M, Garshell J, Neyman N, Altekruse SF Kosary CL, Yu M, Ruhl J, Tatalovich Z, Cho H, Mariotto A, Lewis DR, Chen HS, Feuer EJ, Cronin KA (eds). SEER Cancer Statistics Review, 1975-2010, Bethesda: National Cancer Institute. http://seer.cancer.gov/csr/1975_2010/, based on November 2012 SEER data submission, posted to the SEER web site, April 2013.

3. Allison DC, Carney SC, Ahlmann ER, Hendifar A, Chawla S, Fedenko A, Angeles C, Menendez LR. A meta-analysis of osteosarcoma outcomes in the modern medical era. Sarcoma. 2012;2012:704872.

4. Goorin AM, Schwartzentruber DJ, Devidas M, Gebhardt MC, Ayala AG, Harris MB, Helman LJ, Grier HE, Link MP. Presurgical chemotherapy compared with immediate surgery and adjuvant chemotherapy for nonmetastatic osteosarcoma: Pediatric Oncology Group Study POG-8651. J Clin Oncol Off J Am Soc Clin Oncol. 2003;21(8):1574-80.

5. Kager L, Zoubek A, Potschger U, Kastner U, Flege S, Kempf-Bielack B, Branscheid D, Kotz R, Salzer-Kuntschik M, Winkelmann W, et al. Primary metastatic osteosarcoma: presentation and outcome of patients treated on neoadjuvant Cooperative Osteosarcoma Study Group protocols. J Clin Oncol. 2003;21(10):2011-8.

6. Bacci G, Mercuri M, Longhi A, Ferrari S, Bertoni F, Versari M, Picci P. Grade of chemotherapy-induced necrosis as a predictor of local and systemic control in 881 patients with non-metastatic osteosarcoma of the extremities treated with neoadjuvant chemotherapy in a single institution. Eur J Cancer. 2005; 41(14):2079-85.

7. Hauben El, Weeden S, Pringle J, Van Marck EA, Hogendoorn PC. Does the histological subtype of high-grade central osteosarcoma influence the response to treatment with chemotherapy and does it affect overall survival? A study on 570 patients of two consecutive trials of the European Osteosarcoma Intergroup. Eur J Cancer. 2002;38(9):1218-25.

8. Grimer RJ. Surgical options for children with osteosarcoma. Lancet Oncol. 2005;6(2):85-92.

9. Rasch CR, Hauptmann M, Schornagel J, Wijers O, Buter J, Gregor T, Wiggenraad R, de Boer JP, Ackerstaff AH, Kroger R, et al. Intra-arterial versus 
intravenous chemoradiation for advanced head and neck cancer: Results of a randomized phase 3 trial. Cancer. 2010;116(9):2159-65.

10. Winkler K, Bielack S, Delling G, Salzer-Kuntschik M, Kotz R, Greenshaw C, Jurgens $H$, Ritter J, Kusnierz-Glaz C, Erttmann R, et al. Effect of intraarterial versus intravenous cisplatin in addition to systemic doxorubicin, high-dose methotrexate, and ifosfamide on histologic tumor response in osteosarcoma (study COSS-86). Cancer. 1990;66(8):1703-10.

11. Stewart DJ, Benjamin RS, Zimmerman S, Caprioli RM, Wallace S, Chuang V, Calvo 3rd D, Samuels M, Bonura J, Loo TL. Clinical pharmacology of intraarterial cis-diamminedichloroplatinum(II). Cancer Res. 1983;43(2):917-20.

12. Jaffe N, Knapp J, Chuang VP, Wallace S, Ayala A, Murray J, Cangir A, Wang A, Benjamin RS. Osteosarcoma: intra-arterial treatment of the primary tumor with cis-diammine-dichloroplatinum II (CDP). Angiographic, pathologic, and pharmacologic studies. Cancer. 1983;51(3):402-7.

13. Jaffe N, Raymond AK, Ayala A, Carrasco CH, Wallace S, Robertson R, Griffiths M, Wang YM. Effect of cumulative courses of intraarterial cis-diamminedichloroplatin-II on the primary tumor in osteosarcoma. Cancer. 1989;63(1):63-7.

14. Wilkins RM, Cullen JW, Camozzi AB, Jamroz BA, Odom L. Improved survival in primary nonmetastatic pediatric osteosarcoma of the extremity. Clin Orthop Relat Res. 2005;438:128-36.

15. Hong S, Shin SJ, Jung M, Jeong J, Lee YJ, Shin KH, Roh JK, Rha SY. Comparison of long-term outcome between doublet and triplet neoadjuvant chemotherapy in non-metastatic osteosarcoma of the extremity. Oncology. 2011;80(1-2):107-17.

16. Zhou Y, Huang Z, Wu S, Zang X, Liu M, Shi J. miR-33a is up-regulated in chemoresistant osteosarcoma and promotes osteosarcoma cell resistance to cisplatin by down-regulating TWIST. J Exp Clin Cancer Res. 2014;33:12.

17. Bramwell VH, Burgers M, Sneath R, Souhami R, van Oosterom AT, Voute PA, Rouesse J, Spooner D, Craft AW, Somers R, et al. A comparison of two short intensive adjuvant chemotherapy regimens in operable osteosarcoma of limbs in children and young adults: the first study of the European Osteosarcoma Intergroup. J Clin Oncol Off J Am Soc Clin Oncol. 1992;10(10):1579-91.

18. Bielack SS, Smeland S, Whelan JS, Marina N, Jovic G, Hook JM, Krailo MD, Gebhardt M, Papai Z, Meyer J, et al. Methotrexate, Doxorubicin, and Cisplatin (MAP) Plus Maintenance Pegylated Interferon Alfa-2b Versus MAP Alone in Patients With Resectable High-Grade Osteosarcoma and Good Histologic Response to Preoperative MAP: First Results of the EURAMOS-1 Good Response Randomized Controlled Trial. J Clin Oncol Off J Am Soc Clin Oncol. 2015;33(20):2279-87.

19. Ferrari S, Smeland S, Mercuri M, Bertoni F, Longhi A, Ruggieri P, Alvegard TA, Picci P, Capanna R, Bernini G, et al. Neoadjuvant chemotherapy with high-dose Ifosfamide, high-dose methotrexate, cisplatin, and doxorubicin for patients with localized osteosarcoma of the extremity: a joint study by the Italian and Scandinavian Sarcoma Groups. J Clin Oncol Off J Am Soc Clin Oncol. 2005;23(34):8845-52.

20. Bacci G, Ferrari S, Tienghi A, Bertoni F, Mercuri M, Longhi A, Fiorentini G, Forni C, Bacchini P, Rimondini S, et al. A comparison of methods of locoregional chemotherapy combined with systemic chemotherapy as neoadjuvant treatment of osteosarcoma of the extremity. Eur J Surg Oncol. 2001;27(1):98-104.

21. Bielack S, Kempf-Bielack B, Schwenzer D, Birkfellner T, Delling G, Ewerbeck V, Exner GU, Fuchs N, Gobel U, Graf N, et al. Neoadjuvant therapy for localized osteosarcoma of extremities. Results from the Cooperative osteosarcoma study group COSS of 925 patients. Klin Padiatr. 1999;211(4):260-70.

22. Hugate RR, Wilkins RM, Kelly CM, Madsen W, Hinshaw I, Camozzi AB. Intraarterial chemotherapy for extremity osteosarcoma and MFH in adults. Clin Orthop Relat Res. 2008;466(6):1292-301.

23. McTiernan A, Jinks RC, Sydes MR, Uscinska B, Hook JM, van Glabbeke M, Bramwell V, Lewis IJ, Taminiau AH, Nooij MA, et al. Presence of chemotherapy-induced toxicity predicts improved survival in patients with localised extremity osteosarcoma treated with doxorubicin and cisplatin: a report from the European Osteosarcoma Intergroup. Eur J Cancer. 2012; 48(5):703-12.

24. Tunn PU, Schmidt-Peter P, Pomraenke D, Hohenberger P. Osteosarcoma in children: long-term functional analysis. Clin Orthop Relat Res. 2004;421:212-7.

25. Powers BE, Withrow SJ, Thrall DE, Straw RC, LaRue SM, Page RL, Gillette EL. Percent tumor necrosis as a predictor of treatment response in canine osteosarcoma. Cancer. 1991;67(1):126-34.

26. Petrilli AS, de Camargo B, Filho VO, Bruniera P, Brunetto AL, Jesus-Garcia R, Camargo OP, Pena W, Pericles P, Davi A, et al. Results of the Brazilian
Osteosarcoma Treatment Group Studies III and IV: prognostic factors and impact on survival. J Clin Oncol off J Am Soc Clin Oncol. 2006;24(7):1161-8.

27. Gvozdenovic A, Arlt MJ, Campanile C, Brennecke P, Husmann K, Born W, Muff R, Fuchs B. Silencing of CD44 Gene Expression in Human 143-B Osteosarcoma Cells Promotes Metastasis of Intratibial Tumors in SCID Mice. PLoS One. 2013;8(4):e60329.

28. Arlt MJ, Banke IJ, Walters DK, Puskas GJ, Steinmann P, Muff R, Born W, Fuchs B. LacZ transgene expression in the subcutaneous Dunn/LM8 osteosarcoma mouse model allows for the identification of micrometastasis. J Orthop Res. 2011;29(6):938-46.

29. Husmann K, Arlt MJ, Jirkof P, Arras M, Born W, Fuchs B. Primary tumour growth in an orthotopic osteosarcoma mouse model is not influenced by analgesic treatment with buprenorphine and meloxicam. Lab Anim. 2015; 49(4):284-93.

30. Berndt K, Vogel J, Buehler C, Vogt P, Born W, Fuchs B. A new method for repeated drug infusion into the femoral artery of mice. J Am Assoc Lab Anim Sci. 2012;51(6):825-31.

31. Salzer-Kuntschik M, Brand G, Delling G. Determination of the degree of morphological regression following chemotherapy in malignant bone tumors. Pathologe. 1983;4(3):135-41.

32. Kaufmann SH, Desnoyers S, Ottaviano Y, Davidson NE, Poirier GG. Specific proteolytic cleavage of poly(ADP-ribose) polymerase: an early marker of chemotherapy-induced apoptosis. Cancer Res. 1993;53(17):3976-85.

33. Wang D, Stockard CR, Harkins L, Lott P, Salih C, Yuan K, Buchsbaum D, Hashim A, Zayzafoon M, Hardy RW, et al. Immunohistochemistry in the evaluation of neovascularization in tumor xenografts. Biotech Histochem. 2008;83(3-4):179-89.

34. Jones TW, Chopra S, Kaufman JS, Flamenbaum W, Trump BF. Cis-diamminedichloroplatinum (II)-induced acute renal failure in the rat. Correlation of structural and functional alterations. Lab Invest. 1985; 52(4):363-74.

35. Kuhlmann MK, Burkhardt G, Kohler H. Insights into potential cellular mechanisms of cisplatin nephrotoxicity and their clinical application. Nephrol Dial Transplant. 1997;12(12):2478-80.

36. Harker G, Stephens F. A report on the comparative response of sheep epidermal squamous-cell carcinoma to intraarterial versus intravenous Cisplatin infusion. Int J Oncol. 1995;7(2):365-70.

37. Guijarro MV, Ghivizzani SC, Gibbs CP. Animal models in osteosarcoma. Front Oncol. 2014:4:189

38. Kim B, Kim K, Im KH, Kim JH, Lee JH, Jeon P, Byun H. Multiparametric MR imaging of tumor response to intraarterial chemotherapy in orthotopic xenograft models of human metastatic brain tumor. J Neurooncol. 2016; 127(2):243-51.

39. Ohba T, Cole HA, Cates JM, Slosky DA, Haro H, Ando T, Schwartz HS, Schoenecker JG. Bisphosphonates inhibit osteosarcoma-mediated osteolysis via attenuation of tumor expression of MCP-1 and RANKL. J Bone Mineral Res Off J Am Soc Bone Mineral Res. 2014;29(6):1431-45.

40. Ehrhart N, Eurell JA, Tommasini M, Constable PD, Johnson AL, Feretti A. Effect of cisplatin on bone transport osteogenesis in dogs. Am J Vet Res. 2002;63(5):703-11

41. Elson DA, Thurston G, Huang LE, Ginzinger DG, McDonald DM, Johnson RS, Arbeit JM. Induction of hypervascularity without leakage or inflammation in transgenic mice overexpressing hypoxia-inducible factor-1alpha. Genes Dev. 2001;15(19):2520-32.

42. Mouriaux F, Sanschagrin F, Diorio C, Landreville S, Comoz F, Petit E, Bernaudin M, Rousseau AP, Bergeron D, Morcos M. Increased HIF-1alpha expression correlates with cell proliferation and vascular markers CD31 and VEGF-A in uveal melanoma. Invest Ophthalmol Vis Sci. 2014;55(3):1277-83.

43. Musumeci G, Castorina A, Magro G, Cardile V, Castorina S, Ribatti D. Enhanced expression of CD31/platelet endothelial cell adhesion molecule 1 (PECAM1) correlates with hypoxia inducible factor-1 alpha (HIF-1alpha) in human glioblastoma multiforme. Exp Cell Res. 2015;339(2):407-16.

44. Chiche J, Pommier S, Beneteau M, Mondragon L, Meynet O, Zunino B, Mouchotte A, Verhoeyen E, Guyot M, Pages G, et al. GAPDH enhances the aggressiveness and the vascularization of non-Hodgkin's $B$ lymphomas via NF-kappaB-dependent induction of HIF-1alpha. Leukemia. 2015;29(5):1163-76.

45. Liotta LA, Kleinerman J, Saidel GM. Quantitative relationships of intravascular tumor cells, tumor vessels, and pulmonary metastases following tumor implantation. Cancer Res. 1974;34(5):997-1004.

46. Liotta LA, Saidel MG, Kleinerman J. The significance of hematogenous tumor cell clumps in the metastatic process. Cancer Res. 1976;36(3):889-94. 
47. Nataraj V, Rastogi S, Khan SA, Sharma MC, Agarwala S, Vishnubhatla S, Bakhshi S. Prognosticating metastatic osteosarcoma treated with uniform chemotherapy protocol without high dose methotrexate and delayed metastasectomy: a single center experience of 102 patients. Clin Transl Oncol. 2016.

48. Rasalkar DD, Chu WC, Lee V, Paunipagar BK, Cheng FW, Li CK. Pulmonary metastases in children with osteosarcoma: characteristics and impact on patient survival. Pediatr Radiol. 2011:41(2):227-36.

49. Mizuno T, Taniguchi T, Ishikawa Y, Kawaguchi K, Fukui T, Ishiguro F, Nakamura S, Yokoi K. Pulmonary metastasectomy for osteogenic and soft tissue sarcoma: who really benefits from surgical treatment? Eur J Cardiothorac Surg. 2013;43(4):795-9.

50. van den Hoven AF, Lam MG, Jernigan S, van den Bosch MA, Buckner GD. Innovation in catheter design for intra-arterial liver cancer treatments results in favorable particle-fluid dynamics. J Exp Clin Cancer Res. 2015:34:74.

Submit your next manuscript to BioMed Central and we will help you at every step:

- We accept pre-submission inquiries

- Our selector tool helps you to find the most relevant journal

- We provide round the clock customer support

- Convenient online submission

- Thorough peer review

- Inclusion in PubMed and all major indexing services

- Maximum visibility for your research

Submit your manuscript at www.biomedcentral.com/submit
Biomed Central 